\title{
Effect of Electrical Conductive Property on Electromagnetic Flow Meter
}

\author{
Xiao-Zhang Zhang \\ Department of Engineering Physics, Tsinghua University, Beijing, China \\ zhangxzh@tsinghua.edu.cn
}

\begin{abstract}
:
An electromagnetic flow meter follows Faraday Induction Law. Apart from magnetic field, boundary condition of electrical conductivity is important in picking up the resulting signal. In use of the flow meter, the electrical conductive property of the flow meter may change because of electrode contamination. On the other hand, design of short measuring tube at the cost of losing some inductive voltage is a tendency to make the meter compact. To study these problems, electrodes and measuring tube of the flow meter were modified by insulating painting oil or by aluminum foil. By painting a portion of electrode surface, electrical resistance between two electrodes increased. To simulate a shorter measuring tube, aluminum foil was used to cover part of the inner wall of the tube. A shorter measuring tube would result a reduced inductive voltage in reading. The flow meter with different modifications was tested in a water flow calibration rig for its readings. Variations of readings were calculated and analyzed. In the experiment, it showed that change of electrical conductive property in electrodes caused serious reading errors, while the errors by the change in the meter's tube length seemed to be less. Maximum error in 5 group data of electrode property change was $25 \%$, compared to $10 \%$ of the tube length change in 9 group data.

According to the theory of electromagnetic flow meter, flow at different position in the measuring tube has different contribution to the signal. This is described by the concept of virtual current. Virtual current is in turn determined by electrical boundary condition of the measuring tube as well as electrodes. Any change of these conditions would finally affect the reading of the flow meter. Further, asymmetry of the boundary value also causes error in the reading. The work offered experimental verification for the theory and could help to a better design of the flow meter.
\end{abstract}

Key words: electromagnetic flow meter, electrical conductivity, measuring tube, reading errors

\section{Introduction}

Electromagnetic flow meters work by Faraday's Induction Law. Conducting fluid flowing across a magnetic field will experience electromotive force, which perpendicular both to the flow velocity and the magnetic field[1]. By properly arranging two electrodes, one can obtain a potential difference between the electrodes. This signal is essentially proportional to mean velocity of the flow. Electromagnetic flow meters are widely used in civil water supply, industrial liquids transfer, beverage production lines etc. One of the key advantages of electromagnetic flow meters is unobstructed to the flow. This property makes the flow meters less maintenance and less energy consumption.

During the process of use, suspended substances in fluids will deposit onto the measuring tube of an electromagnetic flow meter, make the electrical property of the tube wall changed. Worse thing would be that the electrodes are contaminated. All these would cause certain output signal error of an electromagnetic flow meter.

To study the effect of electrical conduction change in the measuring tube of an electromagnetic flow meter, we have conducted a number of experiments. Modifications were done on an industrial electromagnetic flow meter. Calibration data were collected to compared with those under normal condition. Results are discussed in light of theory of electromagnetic flow meters.

\section{Experiments}

Experiments were conducted in a standard water flow rate calibration rig. The nominal accuracy of the flow rate rig is $0.3 \%$. The test electromagnetic flow meter is made by Da Gang Instrumentation Ltd of Tian Jin in China. Its nominal diameter is $20 \mathrm{~mm}$, length $176 \mathrm{~mm}$, 
accuracy $0.5 \%$. Calibration is done by timevolume method.

Change of Conductive Condition in Electrodes: To simulate situations of electrode contamination, insulating painting oil was used to cover certain portions of the electrode surfaces. The painted oil was then cleaned using ethanol if necessary to recover the original state. 5 cases were simulated in the experiment. They are shown in Fig. 1

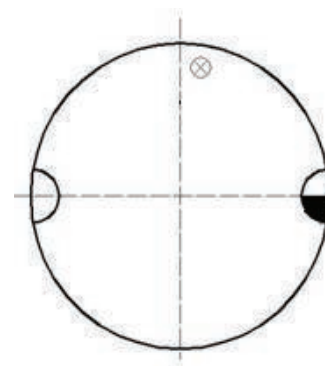

(a)

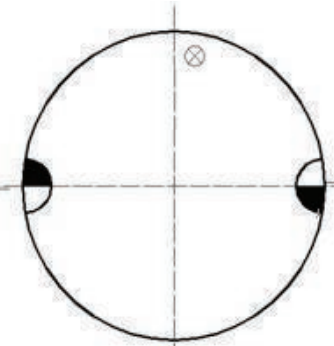

(b)

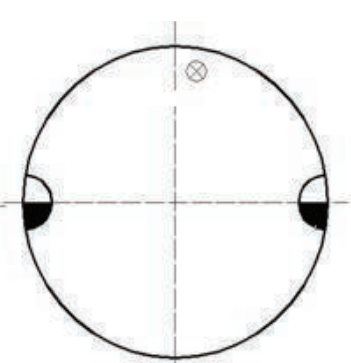

(c)

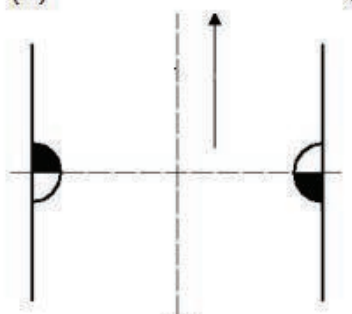

(d)

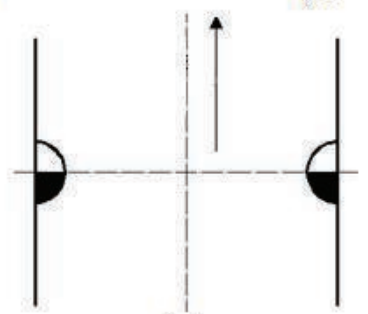

(e)
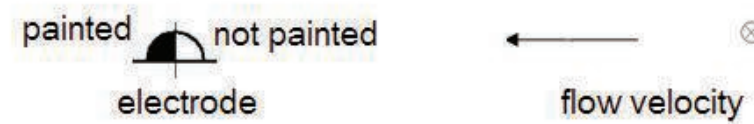

Fig 1 Situations of electrode partially painted by insulating oil (5cases)

After simulating each of the 5 situations of electrode contaminations, calibration was conducted to the modified flow meter. This was done by supply the flow meter with a number of known flow rates, and read the output data from the flow meter. These data were used to calculate errors to the actual flow rates respectively. Actual flow rates were obtained by time-volume method, which is one of standard water flow calibration method. Results were plotted as shown in Figs 2 (a) and (b)

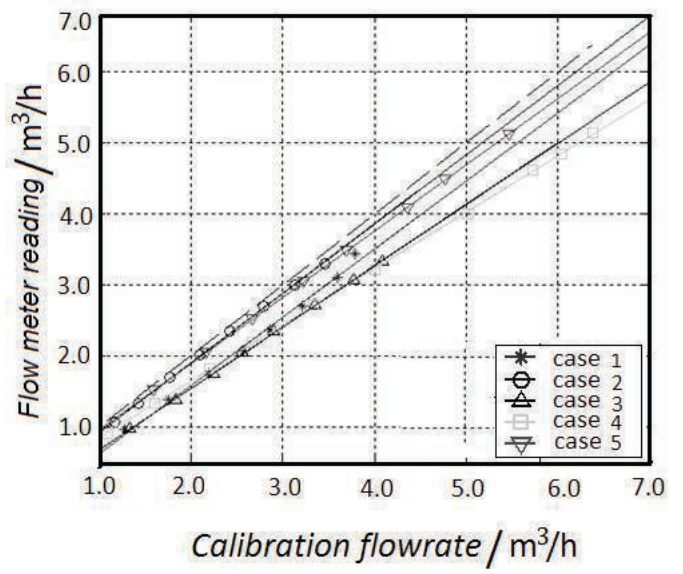

(a) 


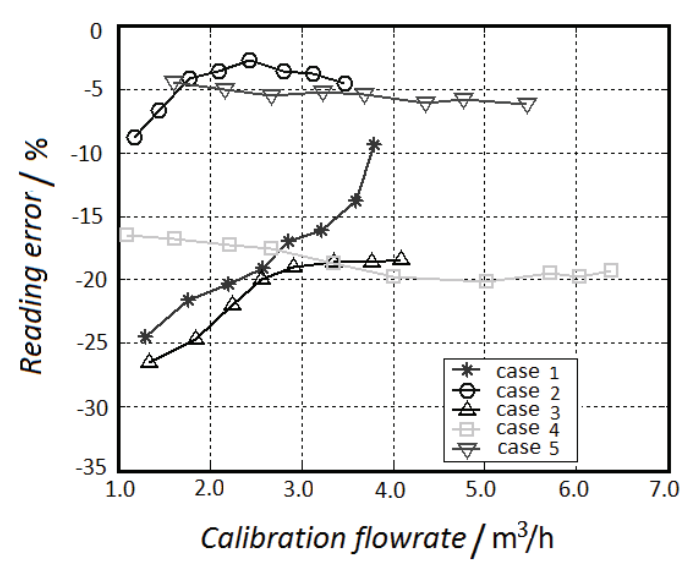

(b)

Fig. 2 Effect of electrode contamination on reading errors of an electromagnetic

From Fig.2(a) one can see that, changes of the electrode conduction seemed not to change the linear relation between the flow rate and the output. However, the changes make quite much reading error in the flow meter as shown in Fig.2(b). Difference between meter's output and actual flow rate is $5 \%$ through $25 \%$ within the experiments. Interestingly, errors in situations of Figs.1(a) (c) and (d) are large than situations in those of Figs1.(b) and (e). The reason possibly comes from asymmetry in formers. In all these cases, the outputs of the flow meter are smaller than actual flow rates respectively. This is because all the cases increase resistance between the electrodes.

Effect of Measuring Tube Length:

In an electromagnetic flow meter, there is an measuring tube where flow acting with magnetic field and inducing induction potential. On the inner wall of the tube, an insulating lining exists. This lining prevents the induction signal from leaking to the earth. Normally the axial length of the lining should be enough long with maximum equal to the tube length. However, tendency of compact design makes the length of the measuring tube become shorter and shorter. Shorter tube means shorter length of the lining. The result is a weaker signal being obtained even other conditions are same. This decrease is not linear to the lining length. For very short tube, signal will decrease quickly with the length. For longer ( larger than 3 times of the tube diameter), increase of the tube length only affect little output signal. Designers need to make balance between enough signal and short tube. To simulate the effect of the measuring tube length to the output of an electromagnetic flow meter. Aluminum foil was used to stick onto the insulating lining. This made the original axial length of the lining change. This change simulates that of measuring tube length. We obtained a number of lining lengths symmetrically or asymmetrically to the electrode line (the electrode line is a straight line linking the two electrodes). See Fig. 3 for definitions.

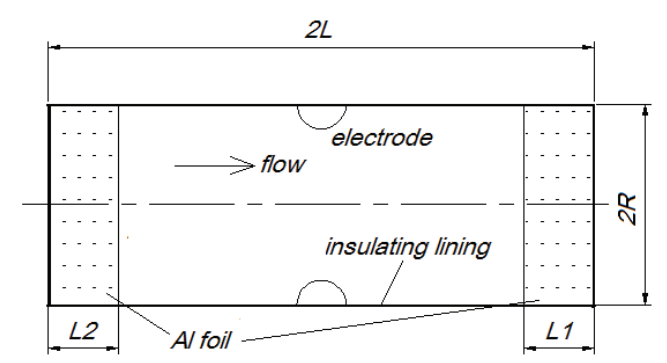

Fig 3 Measuring tube with insulating lining and Aluminum foil

Again, flow rate calibrations were undertaken to the electromagnetic flow meter with changed linings. Reading errors are plotted in Fig. 4.

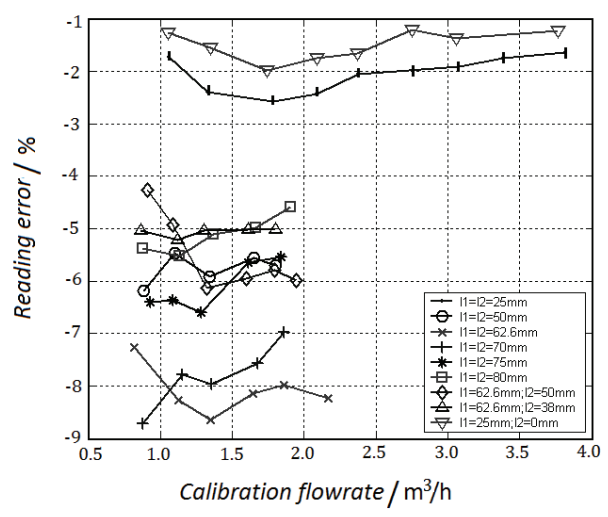

Fig. 4 Effect of measuring tube length on reading errors of an electromagnetic flow meter

In Fig. 4, we can see that change of the lining length causes output signal errors. These errors mainly depend on how much are the changes of the lining length. Basically, errors do not depend on flow rate (just within $2 \%$ ). In 9 cases with different lining lengths, data of error are largely in three groups: the minimum errors of less than $2.5 \%$, the medium $4 \%$ to $6 \%$, the maximum of about $10 \%$. It should be noted that all reading data of flow rates is smaller that actual flow rates respectively.

\section{Discussions}

Above experimental data are the results from simulation of change in electrical conductive property of the flow meter. According to the 
theory of electromagnetic flow meter, output signal of an electromagnetic flow meter depends on three things: its electrical conduction condition (electrodes and insulating lining), magnetic field strength(coils, yoke and power current) and the flow velocity field in the measuring tube[2].

The electrical conductive condition can be explained by a concept of virtual current, A virtual current in an electromagnetic flow meter used to describe the sensitivity of electrodes to flow velocity inside the flow meter tube. It is similar to the electrical current distribution in the tube between the two electrodes with fluid existing. Taking this concept, one can conclude that if any portion of the electrode surface is covered by insulating painting oil, then the virtual current will be weaker because resistance between electrodes increases. A weak virtual current will mean smaller contribution to the output signal. In the same way, if the insulating lining is shorter, more percentage of virtual current will flow into the earth. The result is less virtual current flowing between two electrodes. Then smaller output signal is obtained for the flow meter.

Painting some oil on any of the electrodes will increase resistance of the electrode, while aluminum foil can be taken as perfect conduction. A further problem is where the more sensitive position to the conduction change is. Also, from the concept of virtual current, we can see that virtual current is very dense near the electrodes (especially for point electrodes). This current on an electrode surface increases inversely proportional to the area of the electrode surface. For point electrode, the surface is very small, thus the current here is very dense. Considering any change of the surface area, serious change of the current would occur. However, far away from the electrodes, the virtual current becomes weaker, thus any change of electrical conductivity here would be less affect to the current. Using the concept of virtual current, we can conclude that change of conductive property near the electrodes will cause more sensitive effect to the output signal, while the change of the lining far from the electrodes has less effect. In the experimental data, the change in the electrodes can cause as high as $25 \%$ of measurement error. Reducing the tube length until $1 / 3$ of its original only causes $10 \%$ of the error.

We also tried to make some asymmetrical change of conductive property to the electrode line. For examples, all cases in Fig. 2 and cases 7,8 and 9 in Fig. 4. From the theory of electromagnetic flow meter, asymmetry would cause additional measurement error [3][4]. But it is hard to separate the additional error from total error in the experiment data.

\section{Conclusions}

Main works described in this paper are summarized as bellows:

1) Changes of electrical conductive property in an electromagnetic flow meter are simulated using insulating oil and aluminum foil. Tests were undertaken of the modified flow meter in standard flow rate calibration rig. Output signals were obtained of which 6 cases were electrode changes, 9 cases were insulating lining changes.

2) Experimental results show changes in conductive property on the electrodes surfaces cause up to $25 \%$ of reading errors, while changes on the lining length cause up to $10 \%$ error. In all the cases, output signals are smaller than actual flow rates.

3 ) Concept of virtual current in the theory of electromagnetic flow meter is used to explain to the phenomena, which helps us to obtain an understandable physical figure.

\section{References}

[1] $\mathrm{J}$ Hemp and $\mathrm{H} \mathrm{K}$ Versteeg, Prediction of electromagnetic flowmeter characteristics. J Appl Phys, 19(1986), 1495-76

[2] X-Z Zhang, A method for solving Laplace's equation with mixed boundary condition in electromagnetic flowmeters. J Appl Phys, 22(1989): 573-6

[3] H Shu, H W Lv, X-Z Zhang, Analysis of errors caused by asymmetry of electrodes or coils in electromagnetic flow meter, Measurement Technique, 2005(N.6): 3-4(in Chinese)

[4] GQ Wang, X-Z Zhang, Effect of asymmetrical flow on output of electromagnetic flow meter, Measurement Technique, 2003(N.10): 3-5(in Chinese) 